\title{
Effect of mild restriction of food intake on gene expression profile in the liver of young rats: reference data for in vivo nutrigenomics study
}

\author{
Kenji Saito ${ }^{1,2}$, Yutaka Ohta ${ }^{3}$, Manabu Sami ${ }^{3}$, Tomomasa Kanda ${ }^{3}$ and Hisanori Kato ${ }^{1,2} *$ \\ ${ }^{1}$ Corporate Sponsored Research Program 'Food for Life', Organization for Interdisciplinary Research Projects, The University \\ of Tokyo, 1-1-1 Yayoi, Bunkyo-ku, Tokyo 113-8657, Japan \\ ${ }^{2}$ Department of Applied Biological Chemistry, Graduate School of Agricultural and Life Sciences, The University of Tokyo, \\ 1-1-1 Yayoi, Bunkoyo-ku, Tokyo 113-8657, Japan \\ ${ }^{3}$ Research Laboratories for Health and Gustatory Science, Asahi Breweries Limited, 1-21 Midori 1-Chome, Moriya, Ibaraki \\ 302-0106, Japan
}

(Received 21 January 2010 - Revised 9 March 2010 - Accepted 25 March 2010 - First published online 7 May 2010)

Recent transcriptomics studies on the effect of long-term or severe energy restriction (ER) have revealed that many genes are dynamically modulated by this condition in rodents. The present study was conducted to define the global gene expression profile in response to mild ER treatment. Growing rats were fed with reduced amount of diet (5-30\% ER) for 1 week or 1 month. Using DNA microarray analysis of the liver, seventy-two genes that were consistently changed through the different ER levels were identified. Many were related to lipid metabolism including genes encoding key enzymes such as carnitine palmitoyltransferase 1 and fatty acid synthase. Interestingly, a number of genes were altered even by $5 \%$ ER for 1 week where no differences in weight gain were observed. The information obtained in the present study can be used as a valuable reference data source in the transcriptomics studies of food and nutrition in which subtle differences in food intake sometimes hinder appropriate interpretation of the data.

Energy restriction: Microarrays: Rats: Liver: Nutrigenomics

Energy restriction (ER) prolongs lifespan in a wide variety of organisms, and has been shown to delay the onset or decrease the incidence of many ageing-related diseases, including type 2 diabetes and cancer ${ }^{(1,2)}$. These beneficial effects of ER are likely mediated dynamically through the regulation of a variety of genes ${ }^{(3-5)}$, as has been shown using DNA microarray analyses. Although the gene expression profiles under a dietary-restricted condition have so far been attracting attention as valuable tools for investigating the molecular basis of the ageing process, they can also be highly informative for gaining a broad understanding of the effects of dietary manipulation on animals.

In recent years, DNA microarray technology has been applied to studies on the effects of dietary factors, and has already been successfully utilised in many situations ${ }^{(6,7)}$. In typical studies aimed at elucidating the effect of specific dietary factors, the target component is mix-fed to animals, or the amount or composition of the nutrient under consideration is modified. Such manipulations often result in the change (i.e. decrease in most cases) in the amount of food consumed by the animals or alteration of the diurnal pattern of food intake. These changes might affect the gene expression pattern and hinder appropriate interpretation of gene expression data. In this respect, gene expression profiles under conditions of restriction of food intake will help effectively utilise transcriptomics data in food science when they are used as reference data.

Gene array technology has also offered an approach for screening the ER mimetics, promising preventive agents for ageing-related diseases ${ }^{(8,9)}$. Recently, the commonality of the gene expression pattern between ER and treatment with some anti-diabetic or lipid-lowering agents was reported, suggesting their potency to mimic the ER effect to be manifested at the levels of gene expression ${ }^{(10,11)}$. In this regard, the pattern of gene expression under ER can be used as reference data, which could prove to be a highly potential tool for searching and investigating ER mimetics. Such a strategy can also be applied to nutritional studies, given the growing demands for dietary manipulations leading to ER-like health effects, including the retardation of ageing and the reduction of weight. It has already been established that some dietary components act to reduce the body weight and fat mass of animals without a notable change in food intake ${ }^{(12,13)}$, which resembles one of the major ER-like physiological effects.

The effect of ER on global gene expression has so far been examined in relatively long-term and severe conditions, since the main focus of many ER studies has been its effect on longevity ${ }^{(4,5,14)}$. To our knowledge, there has been no

Abbreviations: ER, Energy restriction; qPCR, quantitative PCR.

* Corresponding author: Dr Hisanori Kato, fax +8135841 1607, email akatoq@mail.ecc.u-tokyo.ac.jp 
comprehensive examination of the gene expression change by subtle dietary restriction such as $10 \%$ ER for a relatively short period, a condition that is similar to that found in the studies of food functionality. Given this fact, the expression data obtained under mild-to-moderate and relatively shortterm ER should be helpful for the effective interpretation of nutrition-related transcriptomics data.

Thus, the present study was conducted to obtain the gene expression profiles of the liver of young growing rodents, one of the most frequently used models in nutritional studies $^{(13,15-17)}$, after mild restriction of food intake $(5-30 \%$ restriction) for 1 week or 1 month.

\section{Materials and methods}

\section{Animal experiment}

Male Wistar rats purchased from Japan SLC (Tokyo, Japan) at 5 weeks of age were housed individually in an air-conditioned room at a controlled temperature of $23 \pm 1{ }^{\circ} \mathrm{C}$ under a $12 \mathrm{~h}$ light-12 $\mathrm{h}$ dark cycle. After a pre-feeding period with an American Institute of Nutrition-93G pellet diet for $3 \mathrm{~d}$ and then with an American Institute of Nutrition-93G powdered diet for 1 week, the rats were divided into ten groups of five each by body weight-balanced randomisation. Two groups and the control groups were given an American Institute of Nutrition-93G powdered diet ad libitum for either 1 week or 1 month. The daily intakes of the control groups were recorded, and 95, 90, 80 and $70 \%$ of the amount consumed by the control groups were given to other groups on the next day; they were designated as 5, 10, 20 and $30 \% \mathrm{ER}$, respectively. Tap water was freely available throughout the experiment. All animal experiments were performed in accordance with the guidelines of the Animal Usage Committee of the Faculty of Agriculture, the University of Tokyo, and were verified by the committee (permission number 1818T0011). Body weight was measured every morning. On the last day of the experiment, after overnight deprivation of food $(16 \mathrm{~h})$, the rats were deeply anaesthetised with diethyl ether and killed by exsanguination of the aorta abdominalis. The liver was then extracted, weighed and stored immediately in RNAlater (Ambion, Tokyo, Japan) solution to stabilise and protect the cellular RNA in intact unfrozen samples. Adipose tissues were also removed and weighed.

\section{Microarray experiments}

Total RNA was isolated from the liver tissues using an RNeasy mini kit with DNase treatment (Qiagen, Valencia, CA, USA) and a QIAshredder (Qiagen) according to the manufacturer's protocol, and was eluted in RNase-free water at a concentration of $1 \mu \mathrm{g} / \mu \mathrm{l}$. An equal amount of RNA from five animals in the same treatment group was pooled and reverse transcribed to the first-strand complementary DNA using SuperScript II RT. Second-strand complementary DNA synthesis was then carried out using a DNA polymerase. Biotinylated complementary RNA was generated from these complementary DNA using a BioArray HighYield RNA transcript labeling kit (Enzo Life Sciences, Farmingdale, NY, USA) according to the standard Affymetrix protocols. It was then purified with RNeasy spin columns (Qiagen), and fragmented before hybridisation.
A pool of complementary RNA was divided in half and used separately for the hybridisation to two GeneChips. Hybridisation mixtures were boiled at $99^{\circ} \mathrm{C}$, loaded on an Affymetrix GeneChip Rat Expression Set 230 Array and hybridised at $45^{\circ} \mathrm{C}$ for $16 \mathrm{~h}$. Washes were performed on a Fluidics Station 450 using the manufacturer's (Affymetrix, Santa Clara, CA, USA) recommended wash solutions, and the chips were stained with a streptavidin-phycoerythrin conjugate for fluorescence detection. After staining, the chips were scanned with an Affymetrix GeneArray Scanner 3000.

\section{Analysis of microarray data}

Image analysis was performed using the Affymetrix GeneChip Operating Software (GCOS version 1.3), which uses the MAS5 (Microarray Suite version 5) algorithm to analyse the scanned images, to obtain a detection call. MAS5 is the official algorithm supplied by Affymetrix, and is used for converting intensities to a numerical format. The detection call indicated whether a transcript was reliably detected (designated as PRESENT) or not detected (designated as ABSENT). A detection $P$ value which is calculated using the one-sided Wilcoxon signed rank test reflects the confidence of the detection call.

The pairwise comparisons were done using GeneChip Operating Software for expression profiles between the ER and control groups, which were designated as baseline arrays. During comparison analysis, each probe set on the experimental array was compared to its counterpart on the baseline array. As a result, the change in $P$ value for 15923 probe sets corresponding to the change call ('increase', 'marginal increase', 'decrease', 'marginal decrease' and 'no change') was obtained. In the present study, we used the default criteria of the GeneChip Operating Software/MAS5's 'detection $P$ value' and 'change $P$ value' which are defined as follows: PRESENT, $P<0.04$; MARGINAL, $0.04 P<0.06$; ABSENT, $P \geq 0.06$; increase, $P \leq 0.0025$; marginal increase, $0.0025<P \leq 0.003$; decrease, $P \geq 0.998$; marginal decrease, $0.997 \leq P<0.998$, and no change, $0.003<P<0.997$. These flags with the above-mentioned criteria are generally used for the determination of differentially expressed genes ${ }^{(18-21)}$ in the Affymetrix GeneChip experiments. Another algorithm was used to calculate a quantitative estimate of the gene expression change in the form of the signal log ratio. In order to figure out dynamic expression changes induced by the mild-to-moderate ER, we did not apply a fold-change cut-off. A further description of the statistical algorithms used here is available at the Affymetrix official site http:// www.affymetrix.com/

To reduce false positives as much as possible, we used a conservative approach in the analysis with a combination of stringent filtering methods. Initially, we excluded the probe sets that were 'ABSENT' in at least one of each hybridisation pair. Then, comparisons with a 'no change' and a 'marginal increase' and a 'marginal decrease' call were removed. We used duplicate GeneChips on each group sample, and the expression change was taken as informative if the change call of both chips was either 'increase' or 'decrease', and was in the same direction. For the gene ontology analysis, all the probe sets with the informative changes were used. Of these genes, the probe sets exhibiting alterations across all the levels of ER in the same direction were considered to be 
Table 1. Body weight gain and organ weight change of rats fed the energy restriction (ER) diet (Mean values with their standard errors, $n 5$ )

\begin{tabular}{|c|c|c|c|c|c|c|c|c|c|c|}
\hline & \multicolumn{2}{|c|}{ Control } & \multicolumn{2}{|c|}{$5 \%$} & \multicolumn{2}{|c|}{$10 \%$} & \multicolumn{2}{|c|}{$20 \%$} & \multicolumn{2}{|c|}{$30 \%$} \\
\hline & Mean & SE & Mean & SE & Mean & SE & Mean & SE & Mean & SE \\
\hline \multicolumn{11}{|l|}{1 week } \\
\hline Body weight gain (g) & $42 \cdot 6$ & 1.5 & $36 \cdot 4$ & 3.1 & $31 \cdot 7^{*}$ & 1.5 & $21 \cdot 1^{\star *}$ & $2 \cdot 7$ & $15 \cdot 1^{\star *}$ & 1.5 \\
\hline Liver weight (g) & $8 \cdot 3$ & 0.2 & $6 \cdot 6^{\star *}$ & 0.2 & $5 \cdot 9^{\star \star}$ & 0.2 & $5 \cdot 2^{\star \star}$ & 0.1 & $4 \cdot 4^{\star \star}$ & 0.1 \\
\hline Epididymal WAT weight (g) & $2 \cdot 0$ & 0.2 & 1.8 & 0.1 & $1 \cdot 7$ & 0.1 & $1.4^{\star *}$ & 0.1 & $1 \cdot 3^{\star *}$ & 0.0 \\
\hline Mesenteric WAT weight (g) & 1.3 & $0 \cdot 1$ & 1.2 & 0.1 & $1 \cdot 2$ & 0.1 & $0.9^{*}$ & 0.2 & $0.8^{\star *}$ & 0.1 \\
\hline Perirenal WAT weight $(\mathrm{g})$ & 1.6 & 0.1 & 1.4 & 0.1 & 1.4 & 0.1 & $1.0^{* *}$ & 0.1 & $0.8^{\star *}$ & 0.0 \\
\hline Total WAT weight (g) & 4.9 & 0.3 & 4.5 & 0.3 & $4 \cdot 3$ & 0.3 & $3 \cdot 2^{\star *}$ & 0.3 & $2 \cdot 9^{\star \star}$ & 0.1 \\
\hline \multicolumn{11}{|l|}{1 month } \\
\hline Body weight gain (g) & $132 \cdot 2$ & 5.5 & $106 \cdot 3^{\star *}$ & 5.7 & $96 \cdot 4^{* *}$ & $2 \cdot 7$ & $89.1^{* *}$ & $2 \cdot 8$ & $69 \cdot 1^{\star *}$ & 1.6 \\
\hline Liver weight (g) & $10 \cdot 9$ & 0.6 & $8 \cdot 2^{\star *}$ & 0.3 & $7 \cdot 6^{\star *}$ & 0.2 & $6 \cdot 9^{\star *}$ & 0.2 & $5 \cdot 7^{\star *}$ & 0.1 \\
\hline Epididymal WAT weight (g) & $5 \cdot 0$ & 0.6 & $6 \cdot 2$ & 0.5 & $5 \cdot 3$ & 0.3 & $4 \cdot 3$ & 0.4 & $3 \cdot 1^{*}$ & 0.2 \\
\hline Mesenteric WAT weight (g) & $2 \cdot 6$ & 0.2 & $2 \cdot 4$ & 0.1 & $2 \cdot 3$ & 0.2 & $1.9^{*}$ & 0.1 & $1 \cdot 3^{\star \star}$ & 0.1 \\
\hline Perirenal WAT weight $(\mathrm{g})$ & $5 \cdot 0$ & 0.6 & 5.4 & 0.2 & 4.6 & 0.2 & 3.9 & 0.2 & $2 \cdot 3^{\star *}$ & 0.1 \\
\hline Total WAT weight (g) & $12 \cdot 6$ & 1.4 & $14 \cdot 0$ & 0.9 & $12 \cdot 2$ & 0.6 & $10 \cdot 1$ & 0.6 & $6 \cdot 8^{* *}$ & 0.3 \\
\hline
\end{tabular}

WAT, white adipose tissue.

Mean values were significantly different between ad libitum group and ER group: ${ }^{\star} P<0.05,{ }^{\star *} P<0.01$ (by ANOVA followed by Dunnett's post hoc test).

ER responsive. The data filtering and identification of overlapped probe sets were carried out using Microsoft Excel. All informative gene expression data were registered to our original microarray database ${ }^{(22)}$ accessible at http://nutrigenomics.jp. The raw data set can be accessed at the GEO site http://www. ncbi.nlm.nih.gov/geo/ (accession number GSE18297).

\section{Quantitative PCR}

To validate the expression data of the DNA microarray, quantitative PCR (qPCR) was conducted using the RNA samples obtained from individual rats. Values for each gene were normalised by the values of the corresponding glyceraldehyde3-phosphate dehydrogenase gene, and the ratios of each ER/ control were calculated. We used glyceraldehyde-3-phosphate dehydrogenase gene as an internal standard control because its mRNA level, quantified by qPCR, was not significantly different in all the experimental groups (data not shown). Primers were designed with a web application (PRIMER3), and their sequences are given in Supplementary Table S1, available online only at http://journals.cambridge.org. SYBR Green EX (Takara Bio, Madison, WI, USA) was used on the real-time PCR detection system (Takara Bio) using thin-wall eight-tube strips. The relative amounts of mRNA were normalised to glyceraldehyde-3-phosphate dehydrogenase, and expressed as the fold-change value of the control.

\section{Functional annotation analysis}

Annotation analysis was performed using the functional annotation tool of the Database for Annotation, Visualization and Integrated Discovery 2.1 program $^{(23,24)}$. This tool is a webaccessible application that can be used to pick up enrichment in gene groups corresponding to particular biological functions or categories. All informative genes are used for the analysis in response to each ER level. For the measurements of comparison between each ER treatment and control, Gene Ontology: Biological Process categories that were significantly overrepresented, as determined by Fisher's exact test, were identified.

\section{Statistical analysis}

Data are presented as the means with their standard errors. Statistical significance was assessed using one-way ANOVA followed by Dunnett's post hoc test for multiple comparisons.

\section{Results}

\section{Body and organ weights}

Throughout the experiment, the body weight of the rats of all groups gradually increased. The weight gain was significantly lower in proportion to the ER level in all the ER groups except in the 1-week $5 \%$ ER group (Table 1). Both the liver weight and adipose tissue weight changed similar to the body weight. No significant differences were observed in the weights of the epididymal, perirenal and mesenteric WAT of the 5 and $10 \%$ ER groups compared with the control group. It was noted that the liver weight was significantly reduced in all the ER groups, including the 1-week $5 \%$ ER group.

\section{The number of altered genes}

The number of genes whose expression levels were judged to be altered according to the criteria described in Materials and methods is given in Table 2. In the 1-week groups, the number

Table 2. The number of gene probes affected by mild-to-moderate energy restriction (ER)

\begin{tabular}{crrrrrr}
\hline & $5 \%$ & $10 \%$ & $20 \%$ & $30 \%$ & Overlap* $^{*}$ & Overlapt \\
\hline Increase & & & & & & \\
1 week & 69 & 420 & 613 & 326 & 53 & 33 \\
1 month & 347 & 286 & 384 & 363 & 146 & \\
Decrease & & & & & & 45 \\
1 week & 114 & 168 & 289 & 408 & 76 & \\
1 month & 148 & 168 & 188 & 207 & 81 & \\
\hline
\end{tabular}

* The number of gene probes exhibiting overlapped changes across all the ER levels within the same period.

$\dagger$ The number of gene probes overlapped throughout all the ER groups. The values were determined according to the criteria described in Materials and methods. 
Table 3. Gene categories determined to be significantly over-represented as down-regulated groups by mildto-moderate energy restriction

\begin{tabular}{|c|c|c|c|c|c|c|c|c|}
\hline \multirow[b]{2}{*}{ Gene ontology term } & \multicolumn{4}{|c|}{1 week } & \multicolumn{4}{|c|}{1 month } \\
\hline & $5 \%$ & $10 \%$ & $20 \%$ & $30 \%$ & $5 \%$ & $10 \%$ & $20 \%$ & $30 \%$ \\
\hline Alcohol metabolism & $\downarrow$ & $\downarrow$ & $\downarrow$ & $\downarrow$ & $\downarrow$ & $\downarrow$ & $\downarrow$ & $\downarrow$ \\
\hline Cellular lipid metabolism & $\downarrow$ & $\downarrow$ & $\downarrow$ & $\downarrow$ & $\downarrow$ & $\downarrow$ & $\downarrow$ & $\downarrow$ \\
\hline Cellular metabolism & $\downarrow$ & $\downarrow$ & $\downarrow$ & $\downarrow$ & $\downarrow$ & $\downarrow$ & $\downarrow$ & $\downarrow$ \\
\hline Cholesterol biosynthesis & $\downarrow$ & $\downarrow$ & $\downarrow$ & $\downarrow$ & $\downarrow$ & $\downarrow$ & $\downarrow$ & $\downarrow$ \\
\hline Cholesterol metabolism & $\downarrow$ & $\downarrow$ & $\downarrow$ & $\downarrow$ & $\downarrow$ & $\downarrow$ & $\downarrow$ & $\downarrow$ \\
\hline Lipid biosynthesis & $\downarrow$ & $\downarrow$ & $\downarrow$ & $\downarrow$ & $\downarrow$ & $\downarrow$ & $\downarrow$ & $\downarrow$ \\
\hline Lipid metabolism & $\downarrow$ & $\downarrow$ & $\downarrow$ & $\downarrow$ & $\downarrow$ & $\downarrow$ & $\downarrow$ & $\downarrow$ \\
\hline Steroid biosynthesis & $\downarrow$ & $\downarrow$ & $\downarrow$ & $\downarrow$ & $\downarrow$ & $\downarrow$ & $\downarrow$ & $\downarrow$ \\
\hline Sterol biosynthesis & $\downarrow$ & $\downarrow$ & $\downarrow$ & $\downarrow$ & $\downarrow$ & $\downarrow$ & $\downarrow$ & $\downarrow$ \\
\hline Carboxylic acid metabolism & & $\downarrow$ & $\downarrow$ & $\downarrow$ & $\downarrow$ & $\downarrow$ & $\downarrow$ & $\downarrow$ \\
\hline Metabolism & $\downarrow$ & $\downarrow$ & $\downarrow$ & $\downarrow$ & $\downarrow$ & $\downarrow$ & & $\downarrow$ \\
\hline Organic acid metabolism & & $\downarrow$ & $\downarrow$ & $\downarrow$ & $\downarrow$ & $\downarrow$ & $\downarrow$ & $\downarrow$ \\
\hline Primary metabolism & & $\downarrow$ & $\downarrow$ & $\downarrow$ & $\downarrow$ & $\downarrow$ & $\downarrow$ & $\downarrow$ \\
\hline Steroid metabolism & $\downarrow$ & & $\downarrow$ & $\downarrow$ & $\downarrow$ & $\downarrow$ & $\downarrow$ & $\downarrow$ \\
\hline Sterol metabolism & $\downarrow$ & & $\downarrow$ & $\downarrow$ & $\downarrow$ & $\downarrow$ & $\downarrow$ & $\downarrow$ \\
\hline Biosynthesis & & & $\downarrow$ & $\downarrow$ & $\downarrow$ & $\downarrow$ & $\downarrow$ & $\downarrow$ \\
\hline Glucose metabolism & & $\downarrow$ & $\downarrow$ & $\downarrow$ & $\downarrow$ & $\downarrow$ & & $\downarrow$ \\
\hline Cellular carbohydrate metabolism & & $\downarrow$ & $\downarrow$ & $\downarrow$ & $\downarrow$ & & & $\downarrow$ \\
\hline Hexose metabolism & & & $\downarrow$ & $\downarrow$ & $\downarrow$ & $\downarrow$ & & $\downarrow$ \\
\hline Monosaccharide metabolism & & & $\downarrow$ & $\downarrow$ & $\downarrow$ & $\downarrow$ & & $\downarrow$ \\
\hline
\end{tabular}

The arrows indicate significant over-representation with $P<0.0001$ (Fisher's exact test).

increased in parallel to the increase in ER levels up to $20 \%$, while no further increase was observed in the $30 \%$ ER group. In contrast, the number of genes was not markedly changed in the 1-month ER groups, although the differences in body weight among the groups were more striking than those in body weight among the 1-week feeding groups. Thus, the ER-dependent increase in the altered genes was not observed in this longer feeding condition. A similar tendency was observed when the threshold value of the signal $\log 2$ ratio was set to 0.5 or 1.0 (data not shown).
The number of informative gene probe sets overlapping among all the ER groups of 1-week feeding was fifty-three for an increase and seventy-six for a decrease. Meanwhile, 146 gene probes for an increase and 81 gene probes for a decrease were overlapped among the groups in the 1-month feeding. Of all, nearly half of the detected probe sets that were altered by $5 \%$ ER in 1-week feeding were also changed in 1-month feeding (Table 2). Ninety gene probes, including functionally obscure genes, altered the expression across all the ER groups. Finally, as given in Tables 5 and 6 ,

Table 4. Gene categories determined to be significantly over-represented as up-regulated groups by mild-to-moderate energy restriction

\begin{tabular}{|c|c|c|c|c|c|c|c|c|}
\hline \multirow[b]{2}{*}{ Gene ontology term } & \multicolumn{4}{|c|}{1 week } & \multicolumn{4}{|c|}{1 month } \\
\hline & $5 \%$ & $10 \%$ & $20 \%$ & $30 \%$ & $5 \%$ & $10 \%$ & $20 \%$ & $30 \%$ \\
\hline Carboxylic acid metabolism & $\uparrow$ & $\uparrow$ & $\uparrow$ & $\uparrow$ & $\uparrow$ & $\uparrow$ & $\uparrow$ & $\uparrow$ \\
\hline Cellular lipid metabolism & $\uparrow$ & $\uparrow$ & $\uparrow$ & $\uparrow$ & $\uparrow$ & $\uparrow$ & $\uparrow$ & $\uparrow$ \\
\hline Fatty acid $\beta$-oxidation & $\uparrow$ & $\uparrow$ & $\uparrow$ & $\uparrow$ & $\uparrow$ & $\uparrow$ & $\uparrow$ & $\uparrow$ \\
\hline Fatty acid metabolism & $\uparrow$ & $\uparrow$ & $\uparrow$ & $\uparrow$ & $\uparrow$ & $\uparrow$ & $\uparrow$ & $\uparrow$ \\
\hline Fatty acid oxidation & $\uparrow$ & $\uparrow$ & $\uparrow$ & $\uparrow$ & $\uparrow$ & $\uparrow$ & $\uparrow$ & $\uparrow$ \\
\hline Lipid metabolism & $\uparrow$ & $\uparrow$ & $\uparrow$ & $\uparrow$ & $\uparrow$ & $\uparrow$ & $\uparrow$ & $\uparrow$ \\
\hline Organic acid metabolism & $\uparrow$ & $\uparrow$ & $\uparrow$ & $\uparrow$ & $\uparrow$ & $\uparrow$ & $\uparrow$ & $\uparrow$ \\
\hline Cellular metabolism & & $\uparrow$ & $\uparrow$ & $\uparrow$ & $\uparrow$ & $\uparrow$ & $\uparrow$ & $\uparrow$ \\
\hline Coenzyme metabolism & & $\uparrow$ & $\uparrow$ & $\uparrow$ & $\uparrow$ & $\uparrow$ & $\uparrow$ & $\uparrow$ \\
\hline Metabolism & & $\uparrow$ & $\uparrow$ & $\uparrow$ & $\uparrow$ & $\uparrow$ & $\uparrow$ & $\uparrow$ \\
\hline Primary metabolism & & $\uparrow$ & $\uparrow$ & $\uparrow$ & $\uparrow$ & $\uparrow$ & $\uparrow$ & $\uparrow$ \\
\hline Biosynthesis & & & $\uparrow$ & $\uparrow$ & $\uparrow$ & $\uparrow$ & $\uparrow$ & $\uparrow$ \\
\hline Generation of precursor metabolites and energy & & $\uparrow$ & $\uparrow$ & $\uparrow$ & & $\uparrow$ & $\uparrow$ & $\uparrow$ \\
\hline Amine catabolism & & & $\uparrow$ & $\uparrow$ & & $\uparrow$ & $\uparrow$ & $\uparrow$ \\
\hline Amine metabolism & & & $\uparrow$ & & $\uparrow$ & $\uparrow$ & $\uparrow$ & $\uparrow$ \\
\hline Amino acid catabolism & & & $\uparrow$ & $\uparrow$ & & $\uparrow$ & $\uparrow$ & $\uparrow$ \\
\hline Amino acid metabolism & & & $\uparrow$ & & $\uparrow$ & $\uparrow$ & $\uparrow$ & $\uparrow$ \\
\hline Cofactor metabolism & & $\uparrow$ & $\uparrow$ & $\uparrow$ & & $\uparrow$ & $\uparrow$ & \\
\hline Nitrogen compound catabolism & & & $\uparrow$ & $\uparrow$ & & $\uparrow$ & $\uparrow$ & $\uparrow$ \\
\hline Nitrogen compound metabolism & & & $\uparrow$ & & $\uparrow$ & $\uparrow$ & $\uparrow$ & $\uparrow$ \\
\hline Steroid metabolism & & $\uparrow$ & $\uparrow$ & $\uparrow$ & & $\uparrow$ & & $\uparrow$ \\
\hline
\end{tabular}

The arrows indicate significant over-representation with $P<0.0001$ (Fisher's exact test). 
seventy-two well-characterised genes from seventy-eight gene probes (thirty-three gene probes for an increase and forty-eight gene probes for a decrease, respectively) were screened out, and defined as ER-responsive genes.

\section{The characterisation of energy restriction-responsive genes}

To characterise the global effect of short-term and mild-tomoderate ER, we searched for enriched biological categories of genes in each level of ER group using the functional annotation tool of National Institutes of Health-Database for Annotation, Visualization and Integrated Discovery. Groups of genes related to alcohol metabolism, cholesterol biosynthesis, sterol synthesis and lipid synthesis were categorised as significantly decreased genes using Fisher's exact test in all the ER groups (Table 3). On the other hand, fatty acid $\beta$-oxidation, carboxylic acid metabolism, organic acid metabolism and cellular lipid metabolism classes were categorised as significantly increased classes (Table 4). The category of amino acid catabolism and nitrogen catabolism was detected as significant in the higher ER levels. Functional annotation analysis proved that significant functional classes were mostly the same across all the ER groups.

We next conducted individual analysis of the genes responding to ER. The genes up-regulated or down-regulated across all the ER groups were defined as ER-responsive genes considering that their expressions were markedly altered by a broad range $(5-30 \%, 1$ week and 1 month) of ER. Moreover, the changes in them were observed even in the 1-week ER treatment, where no body weight change was observed. For these reasons, we focused on characterising these seventy-two genes, which are listed in Tables 5 and 6.

In line with the results obtained through the Database for Annotation, Visualization and Integrated Discovery analysis, many key factors for the regulation of lipid metabolism were found to be highly regulated by screening using our criteria. Most notable gene among them was that related to the downregulation of sterol regulatory element-binding protein 1 , a master regulator of lipid synthesis in the liver of mammals. In concert with this change, lipogenic enzymes such as farnesyl diphosphate synthase, fatty acid synthase, malic enzyme, ATP citrate lyase and acyl-CoA synthetase short-chain family member 2 were down-regulated. On the other hand, genes involved in fatty acid oxidation in the liver such as carnitine palmitoyltransferase-1, which esterifies the long-chain fatty acids to carnitine, and carnitine palmitoyltransferase-2, which releases the fatty acids from carnitine at the inner mitochondrial membrane, were up-regulated in response to ER. Also of note is the elevated expression of the genes for Cyp4A14, a key cytochrome $\mathrm{P} 450$ enzyme catalysing the oxidation of fatty acids.

Table 5. The genes up-regulated across all the energy restriction groups

\begin{tabular}{|c|c|c|c|c|c|c|c|c|c|}
\hline \multirow[b]{2}{*}{ Identifier } & \multicolumn{4}{|c|}{1 week } & \multicolumn{4}{|c|}{1 month } & \multirow[b]{2}{*}{ Gene title } \\
\hline & $5 \%$ & $10 \%$ & $20 \%$ & $30 \%$ & $5 \%$ & $10 \%$ & $20 \%$ & $30 \%$ & \\
\hline 1371012_at & 1.9 & $2 \cdot 2$ & 2.9 & 3.7 & 1.9 & $2 \cdot 4$ & $2 \cdot 8$ & $2 \cdot 5$ & 2-Hydroxyphytanoyl-CoA lyase \\
\hline 1387022_at & $2 \cdot 4$ & 4.4 & $4 \cdot 0$ & $3 \cdot 6$ & $2 \cdot 0$ & $1 \cdot 7$ & $1 \cdot 6$ & 1.4 & Aldehyde dehydrogenase family 1 , member $\mathrm{A} 1$ \\
\hline 1371805_at & 1.7 & $2 \cdot 1$ & $2 \cdot 1$ & 1.9 & 1.6 & 1.7 & 1.4 & $1 \cdot 2$ & Ankyrin repeat domain 46 \\
\hline 1387856_at & 1.6 & $2 \cdot 1$ & $2 \cdot 5$ & $2 \cdot 5$ & $2 \cdot 4$ & $2 \cdot 1$ & $2 \cdot 0$ & $2 \cdot 1$ & Calponin 3, acidic \\
\hline 1367836_at & $1 \cdot 8$ & $2 \cdot 2$ & $2 \cdot 7$ & $3 \cdot 0$ & $2 \cdot 6$ & $4 \cdot 0$ & 3.4 & $2 \cdot 3$ & Carnitine palmitoyltransferase 1 , liver \\
\hline 1386946_at & 1.7 & $2 \cdot 4$ & $2 \cdot 3$ & $2 \cdot 2$ & $2 \cdot 5$ & 3.0 & $2 \cdot 7$ & $2 \cdot 1$ & Carnitine palmitoyltransferase 1 , liver \\
\hline 1386927_at & $1 \cdot 3$ & 1.7 & 1.7 & $2 \cdot 1$ & $2 \cdot 1$ & $2 \cdot 0$ & $2 \cdot 1$ & 1.9 & Carnitine palmitoyltransferase 2 \\
\hline 1376868_at & $1 \cdot 8$ & $2 \cdot 8$ & 3.6 & 3.5 & $2 \cdot 8$ & $2 \cdot 8$ & $2 \cdot 6$ & $3 \cdot 0$ & Cobl-like 1 \\
\hline 1370397_at & 1.5 & $2 \cdot 0$ & $1 \cdot 7$ & $1 \cdot 6$ & 1.4 & 1.5 & 1.5 & 1.5 & Cytochrome P450, family 4 , subfamily a, polypeptide 14 \\
\hline 1368458_at & $2 \cdot 0$ & 3.4 & $5 \cdot 7$ & $7 \cdot 5$ & $6 \cdot 3$ & $7 \cdot 7$ & $6 \cdot 1$ & $4 \cdot 8$ & Cytochrome P450, family 7 , subfamily a, polypeptide 1 \\
\hline 1369485_at & $1 \cdot 7$ & $2 \cdot 1$ & $2 \cdot 7$ & $3 \cdot 1$ & $2 \cdot 1$ & $2 \cdot 5$ & $2 \cdot 4$ & $2 \cdot 6$ & Cytosolic acetyl-CoA hydrolase \\
\hline 1367659_s_at & 1.7 & $2 \cdot 5$ & 2.9 & 3.4 & $2 \cdot 4$ & $2 \cdot 7$ & $2 \cdot 7$ & 2.5 & Dodecenoyl-CoA $\delta$-isomerase \\
\hline 1368536_at & 1.9 & $2 \cdot 6$ & $2 \cdot 6$ & $2 \cdot 8$ & $2 \cdot 1$ & $2 \cdot 3$ & 1.8 & 1.6 & Ectonucleotide pyrophosphatase/phosphodiesterase 2 \\
\hline 1386885_at & $2 \cdot 1$ & $3 \cdot 2$ & 3.9 & $4 \cdot 0$ & $2 \cdot 5$ & $3 \cdot 0$ & $3 \cdot 0$ & $3 \cdot 0$ & Enoyl CoA hydratase 1, peroxisomal \\
\hline 1387053_at & $1 \cdot 7$ & $2 \cdot 8$ & 3.5 & $3 \cdot 2$ & $1 \cdot 7$ & $1 \cdot 7$ & 1.5 & $2 \cdot 5$ & Flavin-containing monooxygenase 1 \\
\hline 1387156_at & $14 \cdot 4$ & $20 \cdot 4$ & $20 \cdot 4$ & $16 \cdot 6$ & $14 \cdot 9$ & $13 \cdot 9$ & $9 \cdot 8$ & $5 \cdot 1$ & Hydroxysteroid (17- $\beta$ ) dehydrogenase 2 \\
\hline 1373975_at & $2 \cdot 1$ & $5 \cdot 1$ & 5.5 & $6 \cdot 3$ & 1.5 & $1 \cdot 6$ & $1 \cdot 7$ & $2 \cdot 1$ & Indolethylamine $\mathrm{N}$-methyltransferase \\
\hline 1369840_at & $2 \cdot 3$ & $3 \cdot 2$ & 4.9 & $5 \cdot 7$ & $2 \cdot 3$ & $2 \cdot 5$ & $3 \cdot 9$ & 3.9 & Integral membrane transport protein UST4r \\
\hline 1377807_a_at & 1.4 & $1 \cdot 8$ & 2.4 & $2 \cdot 1$ & $1 \cdot 8$ & 1.7 & 1.6 & 1.5 & IQ motif and WD repeats 1 \\
\hline 1398296_at & $1 \cdot 6$ & $2 \cdot 5$ & 2.9 & $2 \cdot 7$ & $2 \cdot 5$ & $2 \cdot 5$ & $2 \cdot 3$ & $2 \cdot 0$ & Membrane-interacting protein of RGS16 \\
\hline 1371237_a_at & $5 \cdot 3$ & $11 \cdot 7$ & $17 \cdot 1$ & $16 \cdot 0$ & $10 \cdot 9$ & $11 \cdot 3$ & $8 \cdot 6$ & $15 \cdot 5$ & Metallothionein 1a \\
\hline 1388271_at & $4 \cdot 6$ & $10 \cdot 9$ & $22 \cdot 6$ & 21.9 & $7 \cdot 0$ & $6 \cdot 5$ & $5 \cdot 5$ & $12 \cdot 6$ & Metallothionein 2A \\
\hline 1387336_at & $2 \cdot 0$ & $5 \cdot 3$ & $13 \cdot 0$ & $13 \cdot 0$ & $7 \cdot 5$ & $6 \cdot 7$ & $6 \cdot 5$ & $9 \cdot 5$ & $N$-acetyltransferase 8 (camello like) \\
\hline 1390430_at & $3 \cdot 6$ & 5.5 & 5.5 & $4 \cdot 6$ & $5 \cdot 7$ & $5 \cdot 3$ & $4 \cdot 8$ & $3 \cdot 1$ & Nuclear receptor subfamily 1 , group D, member 2 \\
\hline 1368797_at & 1.7 & $2 \cdot 4$ & $2 \cdot 7$ & $2 \cdot 8$ & 1.6 & $1 \cdot 7$ & $1 \cdot 7$ & $2 \cdot 7$ & Nuclear receptor subfamily 1 , group I, member 3 \\
\hline 1368213_at & 1.7 & $2 \cdot 7$ & $5 \cdot 7$ & $8 \cdot 3$ & $4 \cdot 1$ & 5.9 & $5 \cdot 3$ & $5 \cdot 5$ & P450 (cytochrome) oxidoreductase \\
\hline 1387109_at & 1.9 & 3.0 & 5.9 & $7 \cdot 0$ & $4 \cdot 8$ & 5.9 & $5 \cdot 7$ & $5 \cdot 1$ & P450 (cytochrome) oxidoreductase \\
\hline 1374287_at & 1.6 & $1 \cdot 7$ & 1.5 & $1 \cdot 8$ & 1.4 & 1.5 & $1 \cdot 3$ & 1.5 & Paladin \\
\hline 1372051_at & $2 \cdot 0$ & $2 \cdot 1$ & 3.7 & $4 \cdot 1$ & 2.9 & $3 \cdot 1$ & $2 \cdot 9$ & $2 \cdot 8$ & Rhomboid domain containing 2 \\
\hline 1369864_a_at & 1.4 & 1.5 & $2 \cdot 3$ & $4 \cdot 1$ & $3 \cdot 1$ & $5 \cdot 1$ & $4 \cdot 3$ & 3.9 & Serine dehydratase \\
\hline 1372715_at & 1.4 & 1.9 & $1 \cdot 8$ & 1.7 & $1 \cdot 7$ & $1 \cdot 7$ & $1 \cdot 8$ & 1.4 & Sideroflexin 1 \\
\hline 1372600_at & 1.6 & 1.9 & $2 \cdot 2$ & $2 \cdot 4$ & $2 \cdot 2$ & 1.9 & $2 \cdot 1$ & $2 \cdot 1$ & Similar to F-box only protein 31 \\
\hline 1389738_at & 1.4 & 1.9 & $2 \cdot 0$ & 1.6 & $2 \cdot 1$ & 1.9 & $1 \cdot 7$ & 1.4 & Uracil-DNA glycosylase \\
\hline
\end{tabular}

Genes given Affymetrix's 'PRESENT' (the detection call indicated whether a transcript was reliably detected) call and 'increase' call compared to control group in all the restriction groups were sorted out. Values are the means of two independent hybridisations derived from the same complementary DNA (fold-change). Gene identifier indicates Affymetrix ID, and detailed information is available at http://www.affymetrix.com 
Table 6. The genes down-regulated across all the energy restriction groups

\begin{tabular}{|c|c|c|c|c|c|c|c|c|c|}
\hline \multirow[b]{2}{*}{ Identifier } & \multicolumn{4}{|c|}{1 week } & \multicolumn{4}{|c|}{1 month } & \multirow[b]{2}{*}{ Gene title } \\
\hline & $5 \%$ & $10 \%$ & $20 \%$ & $30 \%$ & $5 \%$ & $10 \%$ & $20 \%$ & $30 \%$ & \\
\hline 1368189_at & 0.5 & 0.4 & 0.2 & 0.2 & 0.3 & 0.3 & 0.3 & 0.3 & 7-Dehydrocholesterol reductase \\
\hline 1371327_a_at & 0.6 & 0.5 & 0.5 & 0.4 & 0.6 & 0.6 & 0.6 & 0.6 & Actg1 actin, $\gamma$, cytoplasmic 1 \\
\hline 1375944_at & 0.7 & 0.6 & 0.4 & 0.4 & 0.6 & 0.5 & 0.5 & 0.5 & Acyl-CoA synthetase short-chain family member 2 \\
\hline 1390383_at & 0.8 & 0.6 & 0.2 & 0.3 & 0.6 & 0.5 & 0.6 & 0.5 & Adipose differentiation-related protein \\
\hline 1368316_at & 0.6 & 0.6 & 0.4 & 0.3 & 0.4 & 0.4 & 0.4 & 0.4 & Aquaporin-8 \\
\hline 1369664_at & 0.7 & 0.5 & 0.4 & 0.4 & 0.3 & 0.4 & 0.4 & 0.6 & Arginine vasopressin receptor $1 \mathrm{~A}$ \\
\hline 1367854_at & 0.5 & 0.3 & 0.2 & 0.1 & 0.3 & 0.2 & 0.2 & 0.2 & ATP citrate lyase \\
\hline 1377192_a_at & 0.6 & 0.4 & 0.3 & 0.4 & 0.4 & 0.3 & 0.4 & 0.6 & Caseinolytic protease $\mathrm{X}$ (Escherichia coll) \\
\hline 1368826_at & 0.8 & 0.6 & 0.4 & 0.3 & 0.6 & 0.4 & 0.3 & 0.3 & Catechol-O-methyltransferase \\
\hline 1372056_at & 0.7 & 0.5 & 0.3 & 0.2 & 0.4 & 0.3 & 0.3 & 0.3 & CKLF-like MARVEL transmembrane domain containing 6 \\
\hline 1375933_at & 0.7 & 0.7 & 0.5 & 0.4 & 0.6 & 0.6 & 0.6 & 0.4 & Claudin 2 \\
\hline 1389144_at & 0.6 & 0.4 & 0.4 & 0.4 & 0.5 & 0.4 & 0.4 & 0.4 & COMM domain containing 6 \\
\hline 1367979_s_at & 0.8 & 0.6 & 0.4 & 0.4 & 0.6 & 0.4 & 0.5 & 0.4 & Cytochrome P450, subfamily 51 \\
\hline 1370830_at & 0.6 & 0.5 & 0.6 & 0.7 & 0.6 & 0.5 & 0.5 & 0.5 & Epidermal growth factor receptor \\
\hline 1367667_at & 0.6 & 0.5 & 0.3 & 0.2 & 0.5 & 0.4 & 0.4 & 0.3 & Farnesyl diphosphate synthase \\
\hline 1370281_at & 0.4 & 0.2 & 0.2 & 0.2 & 0.6 & 0.3 & 0.5 & 0.2 & Fatty acid-binding protein 5 , epidermal \\
\hline 1367707_at & 0.3 & 0.2 & 0.1 & 0.0 & 0.3 & 0.1 & $0 \cdot 1$ & $0 \cdot 1$ & Fatty acid synthase \\
\hline 1367708_a_at & 0.4 & 0.2 & 0.1 & 0.1 & 0.3 & 0.1 & 0.1 & 0.1 & Fatty acid synthase \\
\hline 1387312_a_at & 0.3 & 0.2 & 0.2 & 0.3 & 0.4 & 0.3 & 0.3 & 0.3 & Glucokinase \\
\hline 1372452_at & 0.5 & 0.5 & 0.2 & 0.2 & 0.4 & 0.3 & 0.4 & 0.5 & Glycerol-3-phosphate acyltransferase, mitochondrial \\
\hline 1367894_at & 0.1 & 0.1 & 0.1 & 0.1 & 0.1 & 0.2 & 0.3 & 0.3 & Insulin-induced gene 1 \\
\hline 1389377_at & 0.6 & 0.5 & 0.3 & 0.3 & 0.5 & 0.4 & 0.5 & 0.7 & Insulin-induced gene 2 \\
\hline 1370928_at & 0.6 & 0.4 & 0.2 & 0.3 & 0.4 & 0.3 & 0.3 & 0.4 & Lipopolysaccharide-induced TNF \\
\hline 1370067_at & 0.6 & 0.5 & 0.4 & 0.4 & 0.7 & 0.5 & 0.8 & 0.4 & Malic enzyme 1 \\
\hline 1370870_at & 0.4 & 0.3 & 0.1 & 0.1 & 0.3 & 0.2 & 0.2 & 0.2 & Malic enzyme 1 \\
\hline 1372342_at & 0.5 & 0.3 & 0.3 & 0.2 & 0.5 & 0.5 & 0.5 & 0.4 & Marveld1 MARVEL domain containing 1 \\
\hline 1386918_a_at & 0.7 & 0.5 & 0.3 & 0.2 & 0.3 & 0.4 & 0.3 & 0.3 & Opioid receptor, $\sigma 1$ \\
\hline 1373312_at & 0.6 & 0.5 & 0.3 & 0.3 & 0.3 & 0.3 & 0.3 & 0.4 & Paroxysmal non-kinesigenic dyskinesia \\
\hline 1371646_at & 0.6 & 0.4 & 0.3 & 0.2 & 0.5 & 0.4 & 0.5 & 0.3 & Phosphogluconate dehydrogenase \\
\hline 1373243_at & 0.6 & 0.4 & 0.3 & 0.2 & 0.4 & 0.3 & 0.3 & 0.3 & Phosphomevalonate kinase \\
\hline 1367691_at & 0.7 & 0.6 & 0.4 & 0.4 & 0.7 & 0.5 & 0.6 & 0.3 & Protein kinase $C, \delta$-binding protein \\
\hline $1375215 \_$x_at & 0.6 & 0.5 & 0.3 & 0.2 & 0.4 & 0.3 & 0.3 & 0.4 & Pyroglutamyl-peptidase I \\
\hline 1368651_at & 0.8 & 0.6 & 0.5 & 0.4 & 0.7 & 0.5 & 0.7 & 0.4 & Pyruvate kinase, liver and erythrocyte \\
\hline 1374524_at & 0.7 & 0.6 & 0.5 & 0.4 & 0.6 & 0.5 & 0.5 & 0.4 & Selenocysteine lyase \\
\hline 1386981_at & 0.6 & 0.6 & 0.4 & 0.3 & 0.6 & 0.5 & 0.5 & 0.6 & $\begin{array}{l}\text { Solute carrier family } 16 \text { (monocarboxylic acid transporters), } \\
\text { member } 1\end{array}$ \\
\hline 1371774_at & 0.7 & 0.5 & 0.5 & 0.5 & 0.6 & 0.6 & 0.6 & 0.7 & Spermidine/spermine $N 1$-acetyltransferase \\
\hline 1371104_at & 0.5 & 0.5 & 0.3 & 0.3 & 0.6 & 0.7 & 0.6 & 0.3 & Sterol regulatory element-binding factor 1 \\
\hline 1388426_at & 0.5 & 0.5 & 0.3 & 0.3 & 0.6 & 0.5 & 0.5 & 0.3 & Sterol regulatory element-binding factor 1 \\
\hline 1372727_at & 0.6 & 0.2 & 0.5 & 0.4 & 0.5 & 0.4 & 0.6 & 0.3 & Suppressor of cytokine signaling 2 \\
\hline 1372136_at & 0.8 & 0.5 & 0.4 & 0.4 & 0.5 & 0.5 & 0.5 & 0.6 & Tetraspanin 14 \\
\hline 1390042_at & 0.6 & 0.5 & 0.5 & 0.5 & 0.6 & 0.6 & 0.6 & 0.6 & Transmembrane protein 140 \\
\hline 1372156_at & 0.7 & 0.6 & 0.5 & 0.5 & 0.7 & 0.7 & 0.6 & 0.5 & Transmembrane protein 97 \\
\hline 1367579_a_at & 0.7 & 0.6 & 0.4 & 0.4 & 0.6 & 0.6 & 0.6 & 0.5 & Tubulin, $\alpha 1$ \\
\hline 1371542_at & 0.8 & 0.6 & 0.4 & 0.4 & 0.5 & 0.5 & 0.5 & 0.5 & Tubulin, $\alpha 4$ \\
\hline 1371390_at & 0.7 & 0.6 & 0.4 & 0.4 & 0.6 & 0.6 & 0.6 & 0.4 & Tubulin, $\beta 2$ \\
\hline 1373718_at & 0.3 & 0.1 & 0.1 & 0.1 & 0.1 & 0.1 & 0.1 & 0.2 & Tubulin, $\beta 2$ \\
\hline 1378100_at & 0.7 & 0.7 & 0.5 & 0.5 & 0.7 & 0.6 & 0.6 & 0.6 & YEATS domain containing 4 \\
\hline
\end{tabular}

CKLF, chemokine-like factor; MARVEL, MAL and related proteins for vesicle trafficking and membrane link; COMM, copper metabolism gene MURR1; YEATS, YNK7, ENL, AF-9, TFIIF small subunit.

Genes given Affymetrix's 'PRESENT' (the detection call indicated whether a transcript was reliably detected) call and 'decrease' call compared to control group in all restriction groups were sorted out. Values are the means of two independent hybridisations derived from the same complementary DNA (fold-change). Gene identifier indicates Affymetrix ID, and detailed information is available at http://www.affymetrix.com

In the cholesterol-metabolising pathway, many genes were regulated towards reduction of cholesterol abundance. CYP51, a cytochrome P450 enzyme involved in the post-squalene phases of cholesterol biosynthesis, was down-regulated. 7-Dehydrocholesterol reductase, the enzyme responsible for the final step in the production of cholesterol, was also highly down-regulated. Cyp7a1, the rate-limiting enzyme in the catabolism of cholesterol into bile acids, was up-regulated.

Genes encoding the key enzymes of glycolysis such as pyruvate kinase and glucokinase were down-regulated. The up-regulation of the serine dehydratase gene, which encodes a key enzyme for the catabolism of serine, a glucogenic amino acid, was also detected.

Regarding detoxification, ER induced the gene expression of metallothionein-I and -II, which are involved in the detoxification of heavy metals and protection against various cellular stresses. Aldehyde dehydrogenase family 1 , member A1, a member of the alcohol degradation enzymes, was also up-regulated.

Genes for several structural proteins such as tubulin $\alpha 1$, tubulin $\alpha 4$, tubulin $\beta 2$ and actin $\gamma$ were shown to be downregulated by ER. 
Quantitative PCR validation for microarray data

qPCR was carried out on eight selected genes (of seventy-two ER-responsive genes) to validate the data of the DNA microarray. The genes included those encoding the major metabolic enzymes such as carnitine palmitoyltransferase 1a, farnesyl diphosphate synthase, malic enzyme 1, ATP citrate lyase, fatty acid synthase and glucokinase, whose functions were described earlier and whose changes were thought to be important for the metabolic alterations accompanying mildto-moderate ER. In addition, nuclear receptor subfamily 1, group I, member 3, also known as constitutive androstane
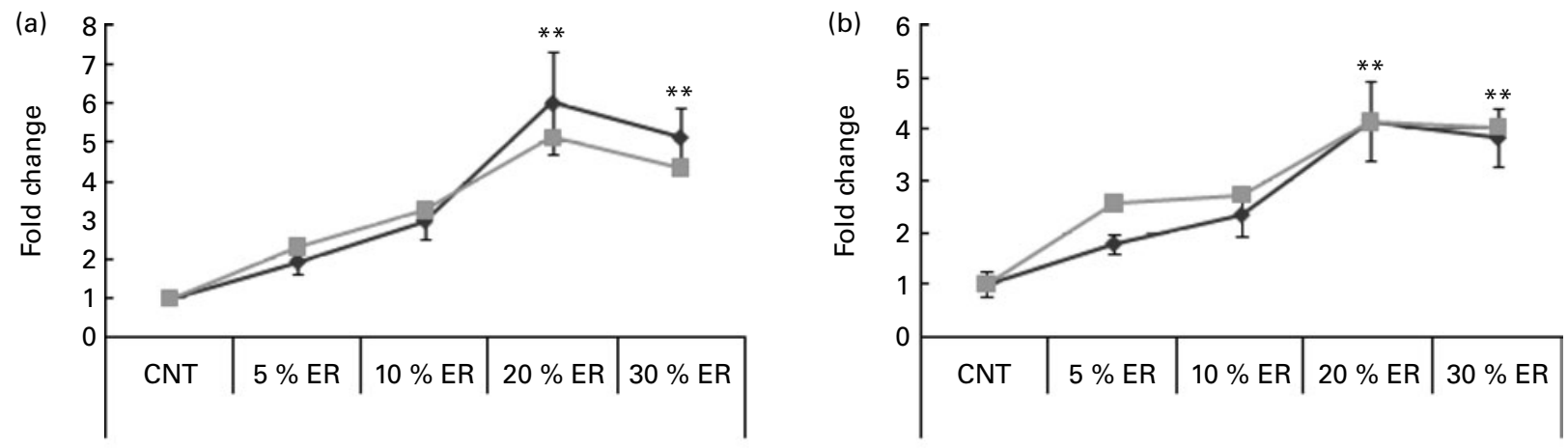

(c) 1.2

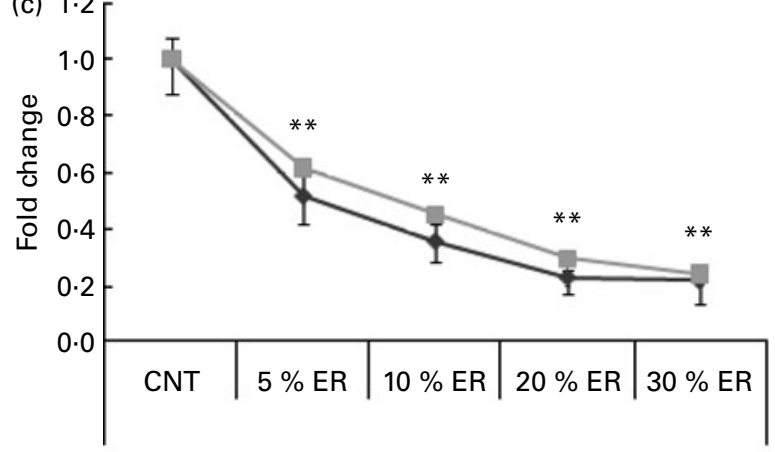

(d) 1
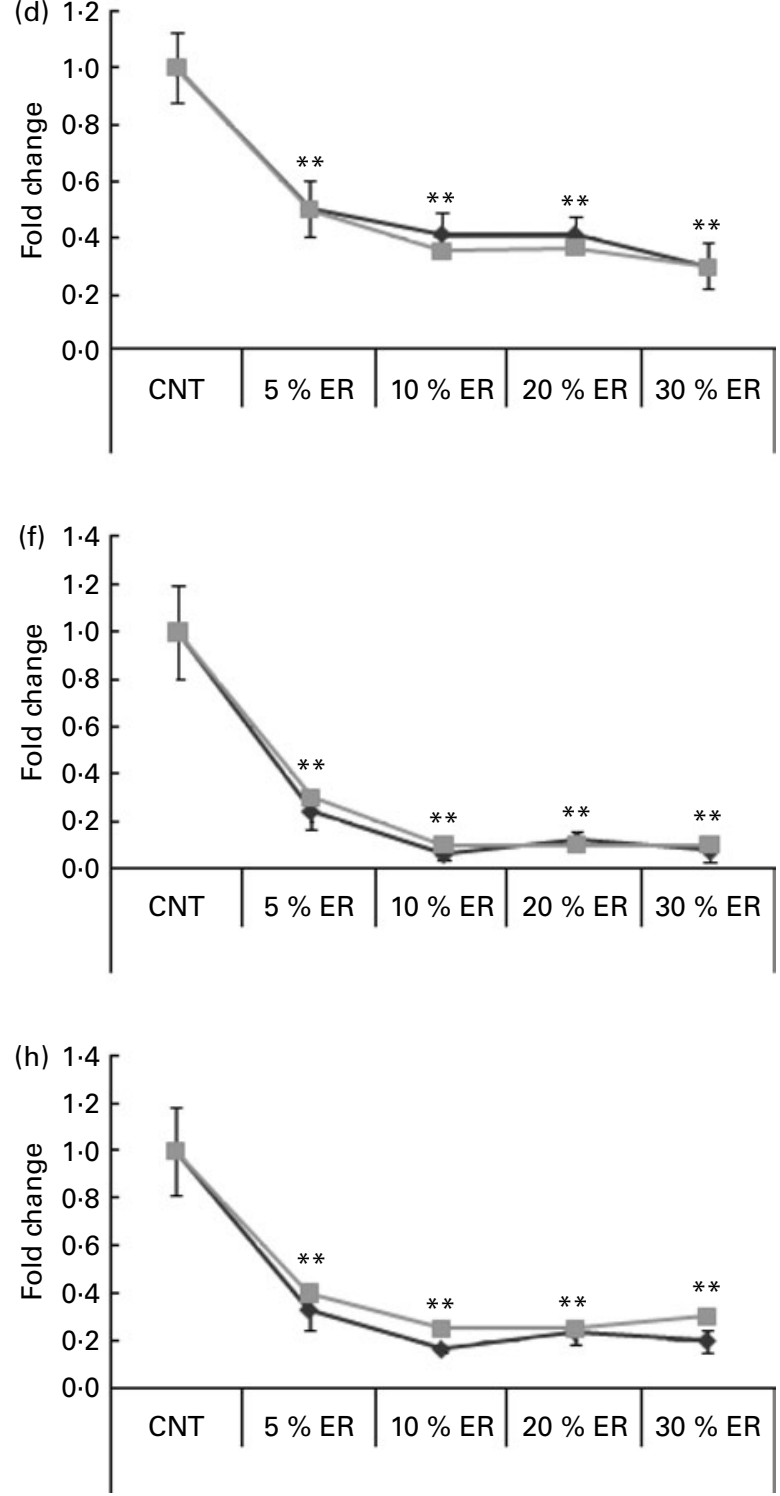

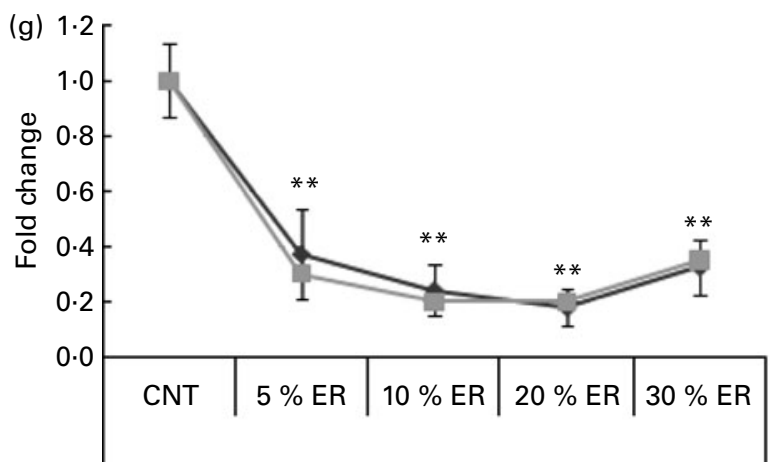



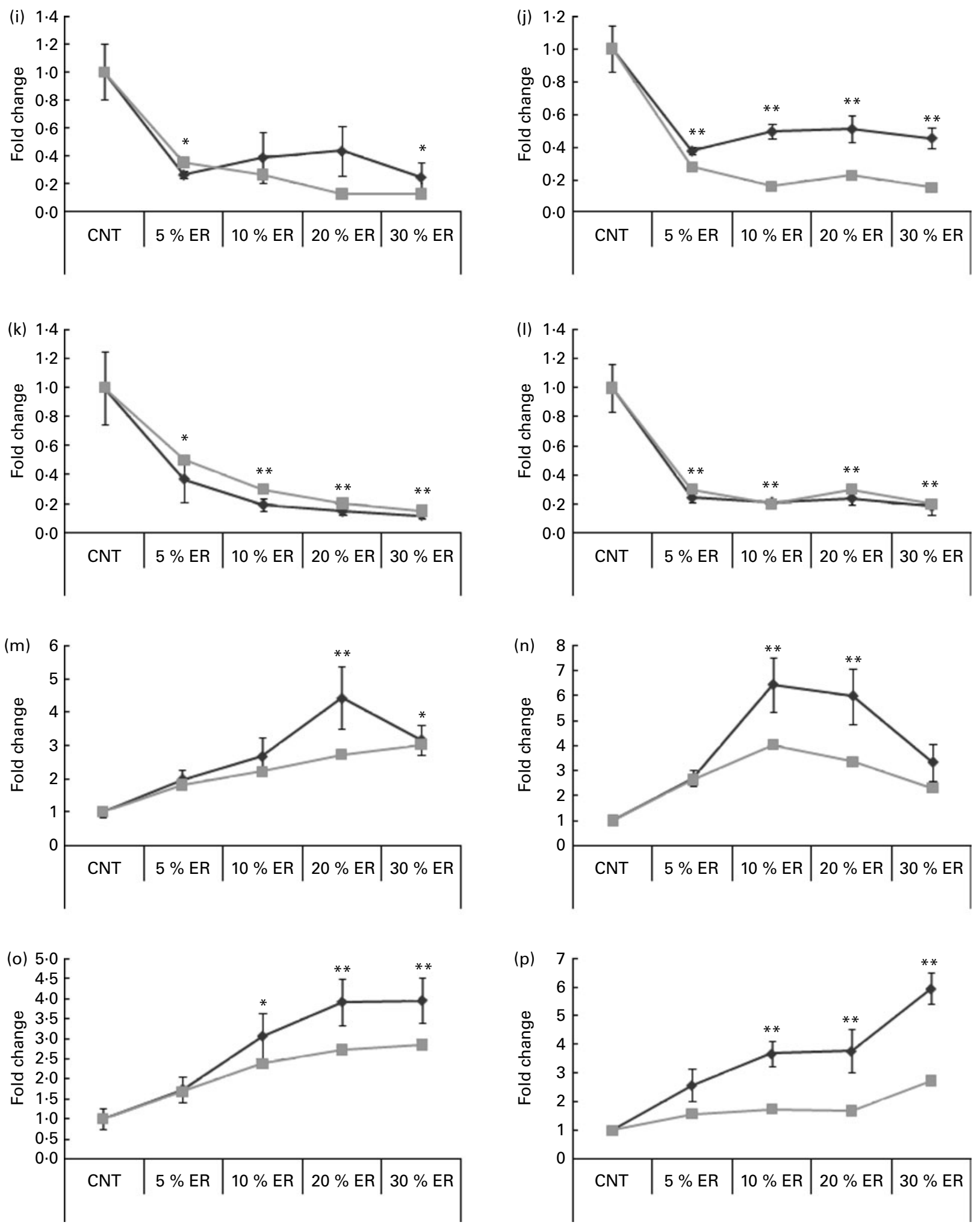

Fig. 1. Validation of microarray data by quantitative PCR (qPCR). Grey lines indicate the values obtained from microarray analysis. Black lines indicate the values obtained from GPCR. Results of QPCR are presented as means with their standard errors of five rats. Mean values were significantly different between ad libitum control (CNT) group and energy restriction (ER) groups: ${ }^{*} P<0.05$, ${ }^{* *} P<0.01$. (a and b) Integral membrane transport protein UST4r (UST4r); (c and d) farnesyl diphosphate synthase (Fdps); (e and f) fatty acid synthase (Fasn); ( $g$ and $h$ ) glucokinase (Gck); ( $i$ and j) malic enzyme 1, NADP(+)-dependent, cytosolic (Me1); (k and I) ATP citrate lyase (Acly); ( $m$ and $n$ ) carnitine palmitoyltransferase 1a, liver (Cpt1a), (o and p) nuclear receptor subfamily 1, group I, member 3 (Nr1i3). a, c, e, g, i, k, m, o: 1 week. b, d, f, h, j, l, n, p: 1 month. 
receptor and shown to be strongly up-regulated in the liver of starved mice ${ }^{(25)}$, was also proven to be highly up-regulated in the present study. The results of the qPCR measurement correlated well with the microarray data, with the correlation coefficient $r^{2}$ values being 0.879 and 0.817 for the 1-week and 1-month treatments, respectively (Supplementary Fig. S1, available online only at http://journals.cambridge.org). Comparison of fold-changes in response to the ER of these eight genes obtained by $\mathrm{qPCR}$ and microarray revealed high consistency in all the cases (Fig. 1). In several genes, the peak response was seen around 10 or $20 \%$ ER in both 1-week and 1-month experiments.

\section{Discussion}

While a number of studies have addressed the effect of ER through the analyses of gene expression profiles in rodents $^{(4,5,14)}$, available information on global gene expression is limited when it comes to low-level and short-term ER treatments. Since the changes in gene expression observed in response to mild ER are likely to reflect sensitive and early events related to the health-promoting and anti-ageing effects of ER, identification of responsive genes by exhaustive analyses should provide a basis for effective utilisation of data, especially those obtained in nutrigenomics research. Specific examples include cases where administration of a food factor affects the amount or temporal pattern of food consumption and/or body weight, which leads to changes in the expression of a group of genes; the responses obtained from experiments of this sort are hard to interpret because they can be attributed to either a direct effect of the food component of interest or indirect effects resulting from alterations of metabolic status. Thus, careful examination of genes that are affected by subtle changes in food consumption should help create an analytical infrastructure for nutritional transcriptomics studies. Therefore, the present study was undertaken to characterise genes that were highly responsive to the mild-to-moderate ER by feeding rats with a slightly reduced amount $(5,10,20$ and $30 \%)$ of food for relatively short periods (1 week and 1 month).

A considerable number of genes were shown to be up- or down-regulated even in the 1 -week $5 \%$ ER group whose body weight gain (and body fat mass) was not significantly different from that of the control group, indicating that the substantial reduction of body weight and fat phenotype is not necessary for the induction of ER-like effects on gene expressions. Among the ER groups, including the 1-week $5 \%$ ER group, a significant loss of liver weight was observed. This reduction may, in part, be involved in the downregulation of several structural proteins given in Table 6 .

Interestingly, the number of responding genes was dependent on the intensity of ER in the 1-week treatment, but not in the 1-month treatment. These differences between the two experimental periods may be derived from a metabolic adaptation in response to the duration of ER. In the course of assessing the reproducibility of microarray data by qPCR, we found that the peak of the gene expression change is located between 10 and $20 \%$ ER in both 1-week and 1-month experiments (Fig. 1). This may account for the fact that the number of genes altered by $30 \%$ ER was smaller than that of genes altered by $20 \%$ ER in the 1-week experiment.
Two types of data analyses, the examination of individual genes and the production of a list of over-represented gene groups by web tool-based annotation analysis, indicated that many genes for lipid biosynthesis were found to be downregulated by ER treatment, while many genes related to fatty acid $\beta$-oxidation were up-regulated. These observations suggest a metabolic shift with ER treatment towards the use of energy, as has been well characterised in previous studies in more severe ER conditions ${ }^{(5)}$. It is also supported by the finding that the representation of the functional annotation category of amino acid catabolism, serving as an important energy source during the fasting state, was significant at a higher level of ER (Table 4). We also observed up-regulation of serine dehydratase, which converts serine to pyruvate. It has been reported that the serine dehydratase pathway is activated in the energy-restricted and starved mouse liver ${ }^{(26,27)}$, indicating the promotion of gluconeogenesis for energy generation. Meanwhile, the repression of glycolysis-related genes such as pyruvate kinase and glucokinase was observed. Moreover, genes related to cholesterol biosynthesis were down-regulated, as was manifested by the annotation analysis also. These results are consistent with the previous reports of the ER effect on the glycolytic and gluconeogenic systems, serum cholesterol and lipid levels ${ }^{(28-32)}$.

On comparing the results obtained in the present study with those obtained in other long-term ER studies ${ }^{(5,33,34)}$, several genes such as metallothionein, carnitine palmitoyltransferase 1, Cyp4A14 and fatty acid synthase were found to be altered to the same direction in at least one of the previous microarray studies examining the effect of ER. Among these genes, Cyp4A14 gene was commonly reported to be altered in the other three studies. By analysing multiple microarray data, Swindell $^{(35)}$ also identified Cyp4A14 as one of the genes upregulated by ER in mouse liver. Cyp4A14 is a downstream target gene of PPAR- $\alpha$, and is involved in the oxidation of fatty acids ${ }^{(36,37)}$. Intriguingly, ER mimetics have also been reported to induce the expression of Cyp4A14 in a PPARdependent manner ${ }^{(11)}$. The fact that Cyp4A14 responded to even very mild conditions such as the 1-week treatment with $5 \%$ ER suggests that this gene could be a candidate marker gene for the PPAR- $\alpha$ activation and ER-like effects. The mild ER-dependent up-regulation of the Cyp4A14 and PPAR- $\alpha$ genes was confirmed by qPCR (data not shown). Combined use of these genes might provide a sensitive probe for the analysis of metabolic status or the screening of ER-mimetic activity.

In conclusion, while most microarray studies on the effect of ER have been undertaken in severe as well as long-term conditions, the present study, conducted to elucidate the gene expression responses to a very subtle level of ER, has revealed that considerable and consistent expression changes are observed in the liver of young rats. The gene expression profile obtained should be highly useful as a reference resource for data mining not only in nutrigenomics studies, but also in pharmaco- and toxicogenomics studies.

\section{Acknowledgements}

We would like to thank Dr Ichiro Matsumoto (ILSI JapanEndowed Chair of Functional Food Science and Nutrigenomics, The University of Tokyo) and Keiko Abe (Graduate 
School of Agricultural and Life Sciences, The University of Tokyo) for their support in conducting the microarray experiments. We also thank Dr Sue-Joan Chang (College of Bioscience and Biotechnology, National Cheng Kung University) for critical reading of the manuscript. This work was supported by the Japan Society for the Promotion of Science (KAKENHI, 18380077). K. S. was the main contributor of the present study in designing the experiments, performing data analysis and preparing the manuscript. Y. O. carried out the data collection. M. S. and T. K. contributed to the interpretation of the data and drafting the report. H. K. coordinated the whole project. There are no conflicts of interest.

\section{References}

1. Jolly CA (2005) Diet manipulation and prevention of aging, cancer and autoimmune disease. Curr Opin Clin Nutr Metab Care 8, 382-387.

2. Bordone L \& Guarente L (2005) Calorie restriction, SIRT1 and metabolism: understanding longevity. Nat Rev Mol Cell Biol 6, 298-305

3. Spindler SR (2005) Rapid and reversible induction of the longevity, anticancer and genomic effects of caloric restriction. Mech Ageing Dev 126, 960-966.

4. Dhahbi JM, Kim HJ, Mote PL, et al. (2004) Temporal linkage between the phenotypic and genomic responses to caloric restriction. Proc Natl Acad Sci U S A 101, 5524-5529.

5. Cao SX, Dhahbi JM, Mote PL, et al. (2001) Genomic profiling of short- and long-term caloric restriction effects in the liver of aging mice. Proc Natl Acad Sci U S A 98, 10630-10635.

6. Kato H, Saito K \& Kimura T (2005) A perspective on DNA microarray technology in food and nutritional science. Curr Opin Clin Nutr Metab Care 8, 516-522.

7. Roy S \& Sen CK (2006) cDNA microarray screening in food safety. Toxicology 221, 128-133.

8. Ingram DK, Anson RM, de Cabo R, et al. (2004) Development of calorie restriction mimetics as a prolongevity strategy. Ann $N$ $Y$ Acad Sci 1019, 412-423.

9. Spindler SR (2006) Use of microarray biomarkers to identify longevity therapeutics. Aging Cell 5, 39-50.

10. Dhahbi JM, Mote PL, Fahy GM, et al. (2005) Identification of potential caloric restriction mimetics by microarray profiling. Physiol Genomics 23, 343-350.

11. Corton JC, Apte U, Anderson SP, et al. (2004) Mimetics of caloric restriction include agonists of lipid-activated nuclear receptors. J Biol Chem 279, 46204-46212.

12. Tachibana N, Matsumoto I, Fukui K, et al. (2005) Intake of soy protein isolate alters hepatic gene expression in rats. J Agric Food Chem 53, 4253-4257.

13. Matsui N, Ito R, Nishimura E, et al. (2005) Ingested cocoa can prevent high-fat diet-induced obesity by regulating the expression of genes for fatty acid metabolism. Nutrition 21, 594-601.

14. Weindruch R, Kayo T, Lee CK, et al. (2001) Microarray profiling of gene expression in aging and its alteration by caloric restriction in mice. J Nutr 131, 918S-923S.

15. Yokohira M, Yamakawa K, Saoo K, et al. (2008) Antioxidant effects of flavonoids used as food additives (purple corn color, enzymatically modified isoquercitrin, and isoquercitrin) on liver carcinogenesis in a rat medium-term bioassay. $J$ Food Sci 73, C561-C568.

16. Abe K, Okada N, Tanabe H, et al. (2009) Effects of chronic ingestion of catechin-rich green tea on hepatic gene expression of gluconeogenic enzymes in rats. Biomed Res 30, 25-29.
17. Sun L, Sadighi Akha AA, Miller RA, et al. (2009) Life-span extension in mice by preweaning food restriction and by methionine restriction in middle age. J Gerontol A Biol Sci Med Sci 64, 711-722.

18. Bardag-Gorce F, Dedes J, French BA, et al. (2007) Mallory body formation is associated with epigenetic phenotypic change in hepatocytes in vivo. Exp Mol Pathol 83, 160-168.

19. Chan MY, Zhao Y \& Heng CK (2008) Sequential responses to high-fat and high-calorie feeding in an obese mouse model. Obesity (Silver Spring) 16, 972-978.

20. Bowers BJ, Radcliffe RA, Smith AM, et al. (2006) Microarray analysis identifies cerebellar genes sensitive to chronic ethanol treatment in PKCgamma mice. Alcohol 40, 19-33.

21. McClintick JN \& Edenberg HJ (2006) Effects of filtering by Present call on analysis of microarray experiments. BMC Bioinformatics 7, 49.

22. Saito K, Arai S \& Kato H (2005) A nutrigenomics database integrated repository for publications and associated microarray data in nutrigenomics research. Br J Nutr 94, 493-495.

23. Dennis G Jr, Sherman BT, Hosack DA, et al. (2003) DAVID: Database for Annotation, Visualization, and Integrated Discovery. Genome Biol 4, P3.

24. Huang da W, Sherman BT \& Lempicki RA (2009) Systematic and integrative analysis of large gene lists using DAVID bioinformatics resources. Nat Protoc 4, 44-57.

25. Bauer M, Hamm AC, Bonaus M, et al. (2004) Starvation response in mouse liver shows strong correlation with lifespan-prolonging processes. Physiol Genomics 17, 230-244.

26. Hagopian K, Ramsey JJ \& Weindruch R (2005) Serine utilization in mouse liver: influence of caloric restriction and aging. FEBS Lett 579, 2009-2013.

27. Snell K (1984) Enzymes of serine metabolism in normal, developing and neoplastic rat tissues. Adv Enzyme Regul 22, 325-400.

28. Dhahbi JM, Mote PL, Wingo J, et al. (1999) Calories and aging alter gene expression for gluconeogenic, glycolytic, and nitrogen-metabolizing enzymes. Am J Physiol 277, E352-E360.

29. Masoro EJ, Compton C, Yu BP, et al. (1983) Temporal and compositional dietary restrictions modulate age-related changes in serum lipids. J Nutr 113, 880-892.

30. Choi YS, Goto S, Ikeda I, et al. (1988) Age-related changes in lipid metabolism in rats: the consequence of moderate food restriction. Biochim Biophys Acta 963, 237-242.

31. Liepa GU, Masoro EJ, Bertrand HA, et al. (1980) Food restriction as a modulator of age-related changes in serum lipids. Am J Physiol 238, E253-E257.

32. Mahoney LB, Denny CA \& Seyfried TN (2006) Caloric restriction in $\mathrm{C} 57 \mathrm{BL} / 6 \mathrm{~J}$ mice mimics therapeutic fasting in humans. Lipids Health Dis 5, 13.

33. Tsuchiya T, Dhahbi JM, Cui X, et al. (2004) Additive regulation of hepatic gene expression by dwarfism and caloric restriction. Physiol Genomics 17, 307-315.

34. Fu C, Hickey M, Morrison M, et al. (2006) Tissue specific and non-specific changes in gene expression by aging and by early stage CR. Mech Ageing Dev 127, 905-916.

35. Swindell WR (2008) Comparative analysis of microarray data identifies common responses to caloric restriction among mouse tissues. Mech Ageing Dev 129, 138-153.

36. Ip E, Farrell GC, Robertson G, et al. (2003) Central role of PPARalpha-dependent hepatic lipid turnover in dietary steatohepatitis in mice. Hepatology 38, 123-132.

37. Reddy JK \& Hashimoto T (2001) Peroxisomal beta-oxidation and peroxisome proliferator-activated receptor alpha: an adaptive metabolic system. Annu Rev Nutr 21, 193-230. 